\title{
Factors associated with breastfeeding cessation in nursing mothers in a peer support programme in Eastern Lancashire
}

Gabriel Agboado ${ }^{1 \dagger}$, Elaine Michel ${ }^{2+}$, Elaine Jackson ${ }^{3+}$, Arpana Verma $^{4^{*+}}$

\begin{abstract}
Background: The UK has one of the lowest breastfeeding rates worldwide and in recent years the Government has made breastfeeding promotion one of its priorities. The UNICEF UK Baby Friendly Initiative is likely to increase breastfeeding initiation but not duration. Other strategies which involve provision of support for breastfeeding mothers in the early weeks after birth are therefore required to encourage UK mothers to breastfeed for the recommended duration. This paper examines the effects of maternal socio-demographic factors, maternal obstetric factors, and in-hospital infant feeding practices on breastfeeding cessation in a peer support setting.

Methods: Data on mothers from Blackburn with Darwen (BwD) and Hyndburn in Eastern Lancashire who gave birth at the Royal Blackburn Hospital and initiated breastfeeding while in hospital were linked to the Index of Multiple Deprivation (IMD). The data were analysed to describe infant feeding methods up to 6 months and the association between breastfeeding cessation, and maternal factors and in-hospital infant feeding practices.

Results: The mean breastfeeding duration was 21.6 weeks (95\% Cl 20.86 to 22.37 weeks) and the median duration was 27 weeks ( $95 \% \mathrm{Cl} 25.6$ to 28.30 weeks). White mothers were $69 \%$ more likely to stop breastfeeding compared with non-White mothers (HR: 0.59; 95\% Cl, 0.52 to 0.67 [White mothers were the reference group]). Breastfeeding cessation was also independently associated with parity and infant feeding practices in hospital. There were no significant associations between breastfeeding cessation and marital status, mode of delivery, timing of breastfeeding initiation and socio-economic deprivation.
\end{abstract}

Conclusion: In this study ethnicity, parity and in-hospital infant feeding practices remained independent predictors of breastfeeding cessation in this peer support setting. However other recognised predictors such as marital status, mode of delivery, timing of breastfeeding initiation and socio-economic deprivation were not found to be associated with breastfeeding cessation.

\section{Background}

The UK has one of the lowest breastfeeding rates worldwide [1] and ranked the second lowest among 32 countries in the WHO European Region with a breastfeeding rate at 6 months of $21 \%$ in 2000 [2]. Only a modest increase was observed by the 2005 Infant Feeding Survey which recorded a breastfeeding rate at 6 months of 25\% [3]. Conversely, some other developed European

\footnotetext{
* Correspondence: Arpana.Verma@manchester.ac.uk

+ Contributed equally

${ }^{4}$ Manchester Urban Collaboration on Health, Manchester Academic Health Sciences Centre, Stopford Building, University of Manchester, Manchester, M13 9PT, UK
}

(C) 2010 Agboado et al; licensee BioMed Central Ltd. This is an Open Access article distributed under the terms of the Creative Commons Attribution License (http://creativecommons.org/licenses/by/2.0), which permits unrestricted use, distribution, and reproduction in any medium, provided the original work is properly cited. high breastfeeding initiation and continuation rates [4].

In recent years the UK Government has made breastfeeding promotion one of its priorities [5-7] and has amended the breastfeeding and weaning recommendations to recommend that infants should be exclusively breastfed for the first 6 months of life [8] following the revision of the WHO guidelines $[9,10]$. Most recently the government proposed the Single Equality Bill under which women would get the legal right to breastfeed in public with the aim of boosting breastfeeding prevalence [11]. Some have observed that the UNICEF UK Baby Friendly Initiative is likely to increase breastfeeding initiation but not duration and suggested that other 
strategies which involve the provision of support for breastfeeding mothers in the early weeks after birth are required to encourage UK mothers to breastfeed for the recommended duration [12].

In 2004 Little Angels, a social enterprise based in Darwen (in Eastern Lancashire), started a collaborative project based on the WHO Ten Steps for Successful Breastfeeding [13] with the Royal Blackburn Hospital, a Baby Friendly Hospital in Lancashire, to support nursing mothers to breastfeed longer. From 2004 to 2006 they helped increase the breastfeeding rates at 6-8 weeks and 7-9 months from $20 \%$ to $56 \%$ and $4 \%$ to $13 \%$ respectively [14]. The initiative received the "Award for practices to promote the involvement of women in providing local maternity services" by the All Party Parliamentary Group (APPG) on Maternity [15]. In the Department of Health's 2007 review of progress against the National Service Framework for Children, Young People and Maternity Services, Little Angels were cited as a case study of good practice [16].

The effectiveness of peer support programmes in improving breastfeeding outcomes has been established [4,17-19]. However breastfeeding duration depends on a number of determinants some of which may be modifiable. Knowledge about the type and importance of these determinants in peer support programmes is essential for building effective breastfeeding promotion programmes [20]. Maternal socio-demographic and obstetric factors such as ethnicity [21-23], parity [24-28], marital status [24-28], mode of delivery [24,25] and socio-economic status $[3,4,24,29]$, and infant feeding practices [13,29-33] are important predictors of breastfeeding cessation. The aim of this study is to identify maternal socio-demographic and obstetric factors, and in-hospital infant feeding practices that are associated with breastfeeding cessation among the mothers supported by Little Angels.

\section{Methods}

\section{The data}

Little Angels run a peer support programme which relies on trained peer support workers. They provide 24-hour telephone support to the nursing mothers in breastfeeding difficulties in addition to regular home visits. Information on feeding methods is collected at 6 weeks, 17 weeks, 6 months, and 9 months. Date of stopping breastfeeding and reasons for stopping are some of the additional information collected.

This study used data from mothers on Little Angels' database and covered all mothers resident in Blackburn with Darwen (BwD) and Hyndburn in East Lancashire who gave birth at the Royal Blackburn Hospital and initiated breastfeeding while in hospital. The database was anonymised and held 2,801 records at the time of the study. Records of mothers with babies aged at least 6 months on $30^{\text {th }}$ May 2007 and with valid postcodes were extracted for analyses. In all 2,107 records were identified meeting these criteria. The main bulk of the data retrieved were for mothers who gave birth between January 2005 and December 2006. Thirteen records were for mothers who gave birth in 2004. Ethical approval was sought from the Cumbria and Lancashire Committee but it was considered not required for this study.

The socio-economic status of the mothers were obtained by linking the data to the 2004 Index of Multiple Deprivation (IMD) [34]. The resultant dataset was analysed to describe infant feeding patterns and the association between breastfeeding cessation, and maternal socio-demographic factors, maternal obstetric factors, and in-hospital infant feeding practices (see table 1 for the list of the factors).

The durations of breastfeeding, in weeks, were calculated from babies' dates of birth and dates of breastfeeding cessation stated by the mothers. Where the dates of breastfeeding cessation were not known the feeding methods at 6 weeks, 17 weeks and 6 months were used to estimate the durations. 1,129 (53.6\%) of the records did not have dates of breastfeeding cessation of which 856 (75.8\%) had entries indicating that the mothers were breastfeeding, at least, up to 6 months.

\section{Outcomes}

This study tested the hypothesis that breastfeeding cessation among the mothers was associated with maternal factors and in-hospital infant feeding practices. The main outcome measures were the mean and median breastfeeding durations in weeks, because the breastfeeding duration data was negatively skewed, and hazard ratios (HRs) of stopping breastfeeding associated with the variables of interest.

The World Health Organization (WHO) has defined three types of breastfeeding namely, exclusive breastfeeding, breastfeeding, and predominant breastfeeding

\section{Table 1 Predictor variables considered for the study}

\begin{tabular}{ll}
\hline Maternal socio-demographic & Borough of residence \\
& Ethnicity \\
& Marital status \\
& Parity \\
& Mode of delivery \\
& Socio-economic deprivation as \\
& measured by the Index of Multiple \\
& Deprivation scores. \\
\hline Infant feeding practices in & The timing of breastfeeding initiation \\
hospital & after delivery \\
& Infant formula-feeding status in \\
& hospital \\
& Teat-giving status in hospital \\
\hline
\end{tabular}


[35]. In this study we used exclusive breastfeeding and breastfeeding as defined by the WHO.

\section{Statistical analyses}

The significance level for all the analyses was set at $5 \%$. Chi-square and Fisher's exact tests were used to test statistical associations for bivariate analyses. Survival analyses techniques were used to estimate the duration of breastfeeding and identify breastfeeding cessation predictors because they provide a good understanding of breastfeeding behaviour over time [27]. KaplanMeier (KM) survival analyses were carried out to describe breastfeeding duration by the variables of interest. To quantify the association between these variables and breastfeeding cessation Cox proportional hazards model was used because it is a more powerful statistical technique than single-point prevalence estimates [27]. To identify variables independently associated with breastfeeding cessation Cox multivariate regression analyses were carried out with all the variables included in the model iteratively using the model analyses based on the likelihood ratio test [36]. Variables which did not significantly contribute to the fitness of the model were dropped until the best model was obtained containing ethnicity, parity, formula-feeding and teats-giving status in hospital as predictor variables. In all 2,074 out of 2,107 records were included in the final model. The results from the final multivariate model were reported as the adjusted HRs. StatsDirect (Version 2.6.3) was used for the analyses.

\section{Results}

Maternal socio-demographic and obstetric characteristics and in-hospital infant feeding practices

Table 2 shows the distribution of maternal socio-demographic and obstetric characteristics. 1,606 (76.2\%) of the mothers were from BwD while 501 (23.8\%) were from Hyndburn. Whites were the predominant ethnicity recorded overall (48.0\%). They constituted $76.0 \%$ among Hyndburn mothers while among BwD mothers they formed $39.1 \%$ ( $p<0.0001)$. More Hyndburn mothers were from areas with IMD scores in the $1^{\text {st }}$ and $2^{\text {nd }}$ quartile (35.9\% and $31.1 \%$ respectively) than those from $\mathrm{BwD}(22.5 \%$ and $22.4 \%$ respectively; $\mathrm{p}<0.0001) .96 .7 \%$ of the mothers had partners, $42.7 \%$ were primiparae and $73.3 \%$ had vaginal deliveries; there were no statistically significant inter-borough differences in the distribution of these factors.

Table 3 shows the distribution of in-hospital infant feeding practices. $77.7 \%$ initiated breastfeeding within one hour after delivery, $76.5 \%$ gave no infant formula, and $84.3 \%$ gave no teats while in hospital. There were no statistically significant inter-borough differences in the distribution of these factors.

\section{Infant feeding methods}

Table 4 shows the distribution of infant feeding methods up to 6 months. Exclusive breastfeeding rates fell as babies grew older. At every assessment point (i.e. 6 weeks, 17 weeks, and 6 months) exclusive breastfeeding rates were higher in $\mathrm{BwD}$ than in Hyndburn. The interborough difference in the rates at 6 weeks was not statistically significant $(\mathrm{p}=0.0594)$ but attained statistical significance at 17 weeks $(48.2 \%$ versus $46.2 \%$; $\mathrm{p}=$ $0.0011)$ and 6 months (14.9\% versus $8.5 \% ; \mathrm{p}=0.0002)$. Mixed breast and formula feeding also declined with increasing age but there were no significant inter-borough differences. Formula feeding peaked at 17 weeks by which time some mothers started introducing weaning foods.

\section{Breastfeeding cessation}

\section{Effects of maternal socio-demographic and obstetric} characteristics on breastfeeding cessation

Table 5 shows breastfeeding durations by maternal factors, and the association between these factors and breastfeeding cessation. KM analyses showed that, overall, the mean breastfeeding duration was 21.6 weeks ( $95 \%$ CI 20.86 to 22.37 weeks) and the median duration was 27 weeks (95\% CI 25.6 to 28.30 weeks). BwD mothers breastfed significantly longer than Hyndburn mothers. The breastfeeding duration for White mothers was significantly shorter than that for non-White mothers. Among non-White mothers Indians and Blacks breastfed longest while Pakistanis had the shortest duration but the differences were not statistically significant.

Partnered mothers breastfed longer than single mothers. The mean and median breastfeeding durations for multiparae were about 2 weeks longer than those for primiparae. Sub-group analyses showed that $2^{\text {nd }}$ born babies were breastfed for the shortest duration while $4^{\text {th }}$ born babies were breastfed for the longest duration. Mothers delivered vaginally breastfed longer than those who had operative deliveries. Sub-group analysis showed that those who had instrumental deliveries breastfed for the shortest duration. However all these differences were not statistically significant.

Unadjusted Cox regression HR showed that Hyndburn mothers were $36 \%$ more likely to stop breastfeeding than BwD mothers (HR: 1.36; 95\% CI, 1.18 to 1.55) but the association became insignificant after adjustment for ethnicity. Unadjusted Cox regression HR showed White mothers were $54 \%$ more likely to stop breastfeeding compared with non-White mothers (HR: 0.65; 95\% CI, 0.57 to 0.73 ). After adjustment White mothers were $69 \%$ more likely to stop breastfeeding compared with nonwhite mothers (HR: 0.59; 95\% CI, 0.52 to 0.67). Subgroup analysis showed that White mothers were $89 \%$ more likely to stop breastfeeding compared with Indian 
Table 2 Distribution of maternal socio-demographic and obstetric characteristics.

\begin{tabular}{|c|c|c|c|c|}
\hline Variable & $\begin{array}{c}\text { Total } \\
\text { No }(\%)\end{array}$ & BwD No (\%) & Hyndburn No (\%) & $P$ value \\
\hline \multicolumn{5}{|l|}{ Borough of residence } \\
\hline Both & 2,107 (100\%) & $1,606(76.2 \%)$ & $501(23.8 \%)$ & - \\
\hline BwD & 1,606 & 1,606 & - & - \\
\hline Hyndburn & 501 & - & 501 & - \\
\hline \multicolumn{5}{|l|}{ Ethnicity } \\
\hline White & $1,003(48.0 \%)$ & $622(39.1 \%)$ & 381 (76.0\%) & $<0.0001$ \\
\hline Non-white & $1,088(52.0 \%)$ & $968(60.9 \%)$ & $120(24.0 \%)$ & $<0.0001$ \\
\hline - Indian & 496 (23.7\%) & 489 (30.8\%) & $7(1.4 \%)$ & $<0.0001$ \\
\hline - Pakistani & $503(24.1 \%)$ & $403(25.3 \%)$ & $100(20.0 \%)$ & 0.0139 \\
\hline - All Blacks & 25 (1.2\%) & $24(1.5 \%)$ & $1(0.2 \%)$ & 0.0097 \\
\hline - Others ${ }^{\Psi}$ & $64(3.1 \%)$ & $52(3.3 \%)$ & $12(2.4 \%)$ & 0.3043 \\
\hline Total valid data & $2,091(100.0 \%)$ & $1,590(100.0 \%)$ & $501(100.0 \%)$ & - \\
\hline Missing data & 16 & 16 & 0 & 0.0094 \\
\hline \multicolumn{5}{|l|}{ Marital status } \\
\hline Mothers with partners & 2,030 (96.7\%) & 1,551 (97.0\%) & 479 (95.8\%) & 0.1557 \\
\hline Single mothers & 69 (3.3\%) & 48 (3.0\%) & 21 (4.2\%) & 0.1557 \\
\hline Total valid data & 2,099 (100.0\%) & 1,599 (100.0\%) & $500(100.0 \%)$ & - \\
\hline Missing data & 8 & 7 & 1 & 0.4498 \\
\hline \multicolumn{5}{|l|}{ IMD quartile } \\
\hline 1st quartile & $542(25.7 \%)$ & $362(22.5 \%)$ & 180 (35.9\%) & $<0.0001$ \\
\hline 2nd quartile & $516(24.5 \%)$ & $360(22.4 \%)$ & 156 (31.1\%) & $<0.0001$ \\
\hline 3rd quartile & $526(25.0 \%)$ & 477 (29.7\%) & 49 (9.8\%) & $<0.0001$ \\
\hline 4th quartile & $523(24.8 \%)$ & 407 (25.3\%) & $116(23.2 \%)$ & 0.3221 \\
\hline Total valid data & $2,107(100.0 \%)$ & $1,606(100.0 \%)$ & $501(100.0 \%)$ & - \\
\hline Missing data & 0 & 0 & 0 & - \\
\hline \multicolumn{5}{|l|}{ Parity } \\
\hline Primips & $897(42.7 \%)$ & $675(42.1 \%)$ & $222(44.3 \%)$ & 0.1952 \\
\hline Multips & 1,206 (57.3\%) & 927 (57.9\%) & $279(55.7 \%)$ & 0.1952 \\
\hline$\cdot 2^{\text {nd }}$ births & $153(7.3 \%)$ & 109 (6.8\%) & $44(8.8 \%)$ & 0.1358 \\
\hline - $3^{\text {rd }}$ births & $521(24.8 \%)$ & $392(24.5 \%)$ & $129(25.7 \%)$ & 0.5580 \\
\hline - $4^{\text {th }}$ births & $296(14.1 \%)$ & $242(15.1 \%)$ & $54(10.8 \%)$ & $0.0152^{\bullet}$ \\
\hline - $5^{\text {th }}$ births & 155 (7.4\%) & 125 (7.8\%) & $30(6.0 \%)$ & 0.1759 \\
\hline - $6^{\text {th }}-9^{\text {th }}$ births & $82(3.9 \%)$ & $60(3.7 \%)$ & $22(4.4 \%)$ & 0.5129 \\
\hline Total valid data & $2,103(100.0 \%)$ & $1,602(100.0 \%)$ & $501(100.0 \%)$ & - \\
\hline Missing data & 4 & 4 & 0 & 0.3112 \\
\hline \multicolumn{5}{|l|}{ Mode of delivery } \\
\hline Vaginal & 1,537 (73.3\%) & 1,183 (74.1\%) & $354(70.8 \%)$ & 0.1333 \\
\hline Operative delivery & $560(26.7 \%)$ & 414 (25.1\%) & $146(29.2 \%)$ & 0.1484 \\
\hline - Caesarean & $371(17.7 \%)$ & $285(17.8 \%)$ & $86(17.2 \%)$ & 0.7377 \\
\hline - Instrumental & 189 (9.0\%) & $129(8.1 \%)$ & $60(12.0 \%)$ & 0.0074 \\
\hline Total valid data & $2,097(100.0 \%)$ & 1,597 (100.0\%) & $500(100.0 \%)$ & - \\
\hline Missing data & 10 & 9 & 1 & 0.2989 \\
\hline
\end{tabular}

"Chinese, Bangladeshi, other ethnic groups constituted "Others". This group is referred to in the text of this paper as other ethnic groups. -Overall Chi-square $p$ was not significant $(p=0.0774)$. 
Table 3 Distribution of in-hospital infant feeding practices.

\begin{tabular}{|c|c|c|c|c|}
\hline Variable & $\begin{array}{c}\text { Total } \\
\text { No }(\%) \\
\end{array}$ & $\begin{array}{c}\text { BwD } \\
\text { No (\%) }\end{array}$ & $\begin{array}{c}\text { Hyndburn } \\
\text { No (\%) }\end{array}$ & $P$ value \\
\hline \multicolumn{5}{|l|}{ Breastfeeding initiation } \\
\hline Within $1 \mathrm{hr}$ & $1,609(77.7 \%)$ & $1,212(77.0 \%)$ & 397 (79.9\%) & 0.1748 \\
\hline After $1 \mathrm{hr}$ & 462 (22.3\%) & $362(23.0 \%)$ & $100(20.1 \%)$ & \\
\hline - After $1 \mathrm{hr}$ to $48 \mathrm{hrs}$ & 419 (20.2\%) & 329 (20.9\%) & $90(18.1 \%)$ & 0.1765 \\
\hline - After 48 hrs & $43(2.1 \%)$ & $33(2.1 \%)$ & $10(2.0 \%)$ & $>0.9999$ \\
\hline Total valid data & $2,071(100.0 \%)$ & $1,574(100.0 \%)$ & $497(100.0 \%)$ & - \\
\hline Missing data & 36 & 32 & 4 & 0.0527 \\
\hline \multicolumn{5}{|l|}{ Formula in hospital } \\
\hline No formula & 1,606 (76.5\%) & $1,212(75.8 \%)$ & $394(78.8 \%)$ & 0.1652 \\
\hline Formula & $493(23.5 \%)$ & $387(24.2 \%)$ & $106(21.2 \%)$ & 0.1652 \\
\hline - Yes (informed choice) & 209 (10.0\%) & $151(9.4 \%)$ & $58(11.6 \%)$ & 0.1472 \\
\hline - Yes (medical indication) & 209 (10.0\%) & $171(10.7 \%)$ & $38(7.6 \%)$ & 0.0404 \\
\hline - Yes (other reasons) & 75 (3.6\%) & 65 (4.1\%) & $10(2.0 \%)$ & 0.0201 \\
\hline Total valid data & 2,099 (100.0\%) & 1,599 (100.0\%) & $500(100.0 \%)$ & - \\
\hline Missing data & 8 & 7 & 1 & 0.4498 \\
\hline \multicolumn{5}{|l|}{ Teats in Hospital } \\
\hline No teats & $1,766(84.3 \%)$ & $1,349(84.6 \%)$ & 417 (83.2\%) & 0.4399 \\
\hline Yes & $329(15.7 \%)$ & $245(15.4 \%)$ & $84(16.8 \%)$ & 0.4399 \\
\hline - Yes (informed choice) & $159(7.6 \%)$ & 95 (6.0\%) & $64(12.8 \%)$ & $<0.0001$ \\
\hline - Yes (other reasons) & $170(8.1 \%)$ & $150(9.4 \%)$ & $20(4.0 \%)$ & $<0.0001$ \\
\hline Total valid data & 2,095 (100.0\%) & $1,594(100.0 \%)$ & $501(100.0 \%)$ & - \\
\hline Missing data & 12 & 12 & 0 & 0.0458 \\
\hline
\end{tabular}

Table 4 Infant feeding methods by borough of residence at 6 weeks, 17 weeks and 6 months.

\begin{tabular}{|c|c|c|c|c|c|c|c|c|c|c|c|c|}
\hline \multirow{2}{*}{$\begin{array}{l}\text { Feeding } \\
\text { methods }\end{array}$} & \multicolumn{4}{|c|}{6 weeks } & \multicolumn{4}{|c|}{17 weeks } & \multicolumn{4}{|c|}{6 months } \\
\hline & $\begin{array}{l}\text { Total } \\
\text { No (\%) }\end{array}$ & $\begin{array}{l}\text { BwD } \\
\text { No (\%) }\end{array}$ & $\begin{array}{l}\text { Hyndburn } \\
\text { No (\%) }\end{array}$ & $\begin{array}{c}P^{\Delta} \\
\text { value }\end{array}$ & $\begin{array}{l}\text { Total } \\
\text { No (\%) }\end{array}$ & $\begin{array}{l}\text { BwD } \\
\text { No (\%) }\end{array}$ & $\begin{array}{l}\text { Hyndburn } \\
\text { No (\%) }\end{array}$ & $\begin{array}{c}\mathrm{P}^{\Delta} \\
\text { value }\end{array}$ & $\begin{array}{l}\text { Total } \\
\text { No (\%) }\end{array}$ & $\begin{array}{l}\text { BwD } \\
\text { No (\%) }\end{array}$ & $\begin{array}{l}\text { Hyndburn } \\
\text { No (\%) }\end{array}$ & $\begin{array}{c}\mathrm{P}^{\Delta} \\
\text { value }\end{array}$ \\
\hline $\begin{array}{l}\text { Exclusively } \\
\text { breast }\end{array}$ & $\begin{array}{c}1,449 \\
(69.2 \%)\end{array}$ & $\begin{array}{c}1,120 \\
(70.3 \%)\end{array}$ & $\begin{array}{c}329 \\
(65.8 \%)\end{array}$ & 0.0594 & $\begin{array}{c}930 \\
(46.2 \%)\end{array}$ & $\begin{array}{c}740 \\
(48.2 \%)\end{array}$ & $\begin{array}{c}190 \\
(39.7 \%)\end{array}$ & 0.0011 & $\begin{array}{c}262 \\
(13.4 \%)\end{array}$ & $\begin{array}{c}223 \\
(14.9 \%)\end{array}$ & $\begin{array}{c}39 \\
(8.5 \%)\end{array}$ & 0.0002 \\
\hline $\begin{array}{l}\text { Exclusively } \\
\text { formula }\end{array}$ & $\begin{array}{c}327 \\
(15.6 \%) \\
\end{array}$ & $\begin{array}{c}235 \\
(14.7 \%) \\
\end{array}$ & $\begin{array}{c}92 \\
(18.4 \%) \\
\end{array}$ & 0.0484 & $\begin{array}{c}414 \\
(20.6 \%) \\
\end{array}$ & $\begin{array}{c}299 \\
(19.5 \%) \\
\end{array}$ & $\begin{array}{c}115 \\
(24.1 \%) \\
\end{array}$ & 0.0212 & $\begin{array}{c}85 \\
(4.3 \%) \\
\end{array}$ & $\begin{array}{c}70 \\
(4.7 \%) \\
\end{array}$ & $\begin{array}{c}15 \\
(3.3 \%) \\
\end{array}$ & 0.1941 \\
\hline $\begin{array}{l}\text { Formula \& } \\
\text { Breast }\end{array}$ & $\begin{array}{c}318 \\
(15.2 \%)\end{array}$ & $\begin{array}{c}239 \\
(15.0 \%)\end{array}$ & $\begin{array}{c}79 \\
(15.8 \%)\end{array}$ & 0.6183 & $\begin{array}{c}258 \\
(12.8 \%)\end{array}$ & $\begin{array}{c}200 \\
(13.0 \%)\end{array}$ & $\begin{array}{c}58 \\
(12.1 \%)\end{array}$ & 0.5853 & $\begin{array}{c}55 \\
(2.8 \%)\end{array}$ & $\begin{array}{c}43 \\
(2.9 \%)\end{array}$ & $\begin{array}{c}12 \\
(2.6 \%)\end{array}$ & 0.7511 \\
\hline Breast \& solid ${ }^{\#}$ & $\begin{array}{c}0 \\
(0.0 \%)\end{array}$ & $\begin{array}{c}0 \\
(0.0 \%)\end{array}$ & $\begin{array}{c}0 \\
(0.0 \%)\end{array}$ & - & $\begin{array}{c}53 \\
(2.6 \%)\end{array}$ & $\begin{array}{c}38 \\
(2.5 \%)\end{array}$ & $\begin{array}{c}15 \\
(3.1 \%)\end{array}$ & 0.3360 & $\begin{array}{c}502 \\
(25.6 \%)\end{array}$ & $\begin{array}{c}392 \\
(26.2 \%)\end{array}$ & $\begin{array}{c}110 \\
(23.9 \%)\end{array}$ & 0.0233 \\
\hline $\begin{array}{l}\text { Formula \& } \\
\text { solid }^{\#}\end{array}$ & $\begin{array}{c}0 \\
(0.0 \%)\end{array}$ & $\begin{array}{c}0 \\
(0.0 \%)\end{array}$ & $\begin{array}{c}0 \\
(0.0 \%)\end{array}$ & - & $\begin{array}{c}4 \\
(0.2 \%) \\
\end{array}$ & $\begin{array}{c}4 \\
(0.3 \%)\end{array}$ & $\begin{array}{c}0 \\
(0.0 \%)\end{array}$ & 0.3111 & $\begin{array}{c}93 \\
(4.7 \%)\end{array}$ & $\begin{array}{c}84 \\
(5.6 \%) \\
\end{array}$ & $\begin{array}{c}9 \\
(2.0 \%)\end{array}$ & 0.0004 \\
\hline $\begin{array}{l}\text { Breast, formula } \\
\& \text { solid" }\end{array}$ & $\begin{array}{c}0 \\
(0.0 \%) \\
\end{array}$ & $\begin{array}{c}0 \\
(0.0 \%) \\
\end{array}$ & $\begin{array}{c}0 \\
(0.0 \%) \\
\end{array}$ & - & $\begin{array}{c}28 \\
(1.4 \%) \\
\end{array}$ & $\begin{array}{c}20 \\
(1.3 \%) \\
\end{array}$ & $\begin{array}{c}8 \\
(1.7 \%) \\
\end{array}$ & 0.3905 & $\begin{array}{c}179 \\
(9.1 \%) \\
\end{array}$ & $\begin{array}{c}130 \\
(8.7 \%) \\
\end{array}$ & $\begin{array}{c}49 \\
(10.6 \%) \\
\end{array}$ & 0.1972 \\
\hline Solid"\# & $\begin{array}{c}0 \\
(0.0 \%)\end{array}$ & $\begin{array}{c}0 \\
(0.0 \%)\end{array}$ & $\begin{array}{c}0 \\
(0.0 \%)\end{array}$ & - & $\begin{array}{c}0 \\
(0.0 \%)\end{array}$ & $\begin{array}{c}0 \\
(0.0 \%)\end{array}$ & $\begin{array}{c}0 \\
(0.0 \%)\end{array}$ & - & $\begin{array}{c}2 \\
(0.1 \%) \\
\end{array}$ & $\begin{array}{c}2 \\
(0.1 \%)\end{array}$ & $\begin{array}{c}0 \\
(0.0 \%)\end{array}$ & - \\
\hline $\begin{array}{l}\text { Had stopped } \\
\mathrm{BF}^{*}\end{array}$ & $\begin{array}{c}0 \\
(0.0 \%)\end{array}$ & $\begin{array}{c}0 \\
(0.0 \%)\end{array}$ & $\begin{array}{c}0 \\
(0.0 \%)\end{array}$ & - & $\begin{array}{c}326 \\
(16.2 \%)\end{array}$ & $\begin{array}{c}234 \\
(15.2 \%)\end{array}$ & $\begin{array}{c}92 \\
(19.2 \%)\end{array}$ & 0.0336 & $\begin{array}{c}781 \\
(39.9 \%)\end{array}$ & $\begin{array}{c}554 \\
(37.0 \%)\end{array}$ & $\begin{array}{c}227 \\
(49.2 \%)\end{array}$ & $\begin{array}{c}< \\
0.0001\end{array}$ \\
\hline $\begin{array}{l}\text { Total valid } \\
\text { data }\end{array}$ & $\begin{array}{c}2,094 \\
(100.0 \%)\end{array}$ & $\begin{array}{c}1,594 \\
(100.0 \%)\end{array}$ & $\begin{array}{c}500 \\
(100.0 \%)\end{array}$ & - & $\begin{array}{c}2,013 \\
(100.0 \%)\end{array}$ & $\begin{array}{c}1,535 \\
(100.0 \%)\end{array}$ & $\begin{array}{c}478 \\
(100.0 \%)\end{array}$ & - & $\begin{array}{c}1,959 \\
(100.0 \%)\end{array}$ & $\begin{array}{c}1,498 \\
(100.0 \%)\end{array}$ & $\begin{array}{c}461 \\
(100.0 \%)\end{array}$ & - \\
\hline Missing data & 13 & 12 & 1 & 0.2081 & 94 & 71 & 23 & 0.8057 & 148 & 108 & 40 & 0.3188 \\
\hline
\end{tabular}

${ }_{\mathrm{s}} \mathrm{p}$ values are for the differences between BwD and Hyndburn mothers.

\#'Solid' refer to weaning food.

* Little Angels stop follow-up visits/contacts with mothers who had stopped breastfeeding at any assessment point. "Had stopped BF" refers to those mothers. 
Table 5 Effects of maternal socio-demographic and obstetric factors on breastfeeding cessation.

\begin{tabular}{|c|c|c|c|c|c|c|}
\hline & Breastfeeding durati & from KM analyses & Unadjusted Cox HR & & Adjusted Cox HR & \\
\hline Variable & Mean $(95 \% \mathrm{Cl})$ & Median $(95 \% \mathrm{Cl})$ & $(95 \% \mathrm{Cl})$ & $P$ value & $(95 \% \mathrm{Cl})$ & $P$ value \\
\hline \multicolumn{7}{|l|}{ Borough of residence } \\
\hline Both & 21.6 (20.86 to 22.37$)$ & 27 (25.70 to 28.30$)$ & - & - & - & - \\
\hline BwD & 22.5 (21.63 to 23.39) & 27 (26.11 to 27.88) & $1^{\dagger}$ & - & - & - \\
\hline Hyndburn & 18.5 (17.36 to 19.73$)$ & 22 (18.69 to 25.30$)$ & 1.36 (1.18 to 1.55$)$ & $<0.0001$ & - & - \\
\hline Ethnicity & & & & & - & - \\
\hline White & 19.8 (18.53 to 20.97) & 20 (17.60 to 22.39) & $1^{\dagger}$ & - & $1^{\dagger}$ & - \\
\hline Non-white & 23.4 (22.42 to 24.34$)$ & 27 (26.03 to 27.97) & 0.65 (0.57 to 0.73$)$ & $<0.0001$ & 0.59 (0.52 to 0.67$)$ & $<0.0001$ \\
\hline - Indian & 24.8 (23.53 to 26.15$)$ & 28 (26.67 to 29.33) & 0.55 (0.46 to 0.64$)$ & $<0.0001$ & 0.53 (0.45 to 0.62 ) & $<0.0001$ \\
\hline - Pakistani & 21.8 (20.27 to 23.25$)$ & 26 (25.05 to 26.95$)$ & 0.79 (0.68 to 0.91$)$ & 0.0015 & 0.69 (0.60 to 0.81$)$ & $<0.0001$ \\
\hline - All Blacks & 25.1 (20.79 to 29.51) & 28 (25.18 to 30.81$)$ & 0.40 (0.19 to 0.84$)$ & 0.0155 & 0.35 (0.16 to 0.74$)$ & 0.0057 \\
\hline - Others $^{\Psi}$ & 24.5 (20.11 to 28.81) & 27 (25.89 to 28.11$)$ & 0.52 (0.35 to 0.78$)$ & 0.0018 & 0.48 (0.32 to 0.73$)$ & 0.0005 \\
\hline \multicolumn{7}{|l|}{ Marital status } \\
\hline Single mothers & $18.2(15.19$ to 21.17$)$ & 24 (17.77 to 30.23$)$ & $1^{\dagger}$ & - & - & - \\
\hline Mothers with partners & 21.6 (20.89 to 22.35$)$ & 27 (25.69 to 28.31$)$ & 0.80 (0.58 to 1.11$)$ & 0.1815 & - & - \\
\hline \multicolumn{7}{|l|}{ IMD quartile } \\
\hline 1st quartile & 21.3 (19.69 to 22.82) & 25 (22.71 to 27.29$)$ & $1^{\dagger}$ & - & - & - \\
\hline 2nd quartile & 20.9 (19.52 to 22.21$)$ & 26 (23.36 to 28.64$)$ & $1.01(0.86$ to 1.19$)$ & 0.8873 & - & - \\
\hline 3rd quartile & 23.2 (21.37 to 24.97 ) & 27 (26.21 to 27.79) & 0.78 (0.66 to 0.92$)$ & 0.0043 & - & - \\
\hline 4th quartile & 20.8 (19.66 to 21.96) & 27 (24.86 to 29.13 ) & $0.90(0.76$ to 1.06$)$ & 0.2097 & - & - \\
\hline \multicolumn{7}{|l|}{ Parity } \\
\hline Primips & 20.4 (19.24 to 21.54) & 25 (23.19 to 26.81$)$ & $1.25(1.11$ to 1.41$)$ & 0.0003 & $1.23(1.09$ to 1.39$)$ & 0.0007 \\
\hline Multips ${ }^{\S}$ & 22.5 (21.54 to 23.50 ) & 27 (25.99 to 28.01) & $1^{\dagger}$ & - & $1^{\dagger}$ & - \\
\hline$\cdot 2^{\text {nd }}$ births & 20.0 (16.71 to 23.35$)$ & 18 (14.21 to21.79) & - & - & - & - \\
\hline - $3^{\text {rd }}$ births & 22.4 (21.05 to 23.80$)$ & 27 (26.12 to 27.88$)$ & - & - & - & - \\
\hline - $4^{\text {th }}$ births & 23.5 (21.55 to 25.36$)$ & 27 (23.98 to 30.02) & - & - & - & - \\
\hline$\cdot 5^{\text {th }}$ births & 22.6 (20.44 to 24.76$)$ & 27 (25.63 to 28.37 ) & - & - & - & - \\
\hline$\cdot 6^{\text {th }}-9^{\text {th }}$ births & 22.4 (20.46 to 24.42 ) & 29 (not calculated) & - & - & - & - \\
\hline $\begin{array}{l}\text { - Mothers with } \mathrm{n}^{\text {th }} \text { birth; } \\
(1 \leq \mathrm{n} \leq 8)\end{array}$ & - & - & $1+$ & - & $1+$ & - \\
\hline - Mothers with $(\mathrm{n}+1)^{\mathrm{th}}$ birth & - & - & 0.92 (0.89 to 0.96$)$ & $<0.0001$ & 0.93 (0.89 to 0.97$)$ & 0.0006 \\
\hline \multicolumn{7}{|l|}{ Mode of delivery } \\
\hline Vaginal & 22.0 (21.15 to 22.88$)$ & 27 (25.72 to 28.28$)$ & $1^{\dagger}$ & - & - & - \\
\hline - Operative delivery & 20.4 (18.95 to 21.92) & 25 (23.15 to 26.85$)$ & 1.16 (1.01 to 1.32$)$ & 0.0307 & - & - \\
\hline - Caesarean & 20.9 (19.10 to 22.71) & 25 (23.18 to 26.82$)$ & $1.13(0.96$ to 1.31$)$ & 0.1336 & - & - \\
\hline - Instrumental & 19.5 (16.88 to 22.03$)$ & 24 (19.67 to 28.32) & $1.23(0.98$ to 1.53$)$ & 0.0523 & - & - \\
\hline
\end{tabular}

$\Phi$ Variables that did not significantly contribute to the fitness of the model were dropped hence no adjusted HRs were calculated for them. ${ }^{\dagger}$ Reference category.

"Chinese, Bangladeshi, other ethnic groups constituted "Others". This group is referred to in the text of this paper as other ethnic groups.

${ }^{s}$ Birth order was entered in the Cox model as a continuous variable hence HRs were not reported for the sub-categories used for KM analyses.

mothers (HR: 0.53 ; 95\% CI, 0.45 to 0.62 ), $45 \%$ more likely to stop compared with Pakistanis (HR: 0.69; 95\% CI, 0.60 to 0.81 ) and 2.9 times more likely to stop compared with Blacks (HR: 0.35; 95\% CI, 0.16 to 0.81 ).

Marital status was not associated with breastfeeding cessation in unadjusted Cox regression analyses (HR: 0.80; $95 \%$ CI, 0.58 to 1.11). From unadjusted Cox regression analyses primiparae were $25 \%$ more likely to stop breastfeeding compared with multiparae (HR: 1.25; 95\% CI, 1.11 to 1.41 ); the association was slightly attenuated after adjustment (HR: 1.23; 95\% CI, 1.09 to 1.39). Replacing parity as a binary variable with birth order in the model showed that mothers with babies of higher order birth were less likely to stop breastfeeding than those with lower birth order babies. For example mothers with $3^{\text {rd }}$ born babies were about $8 \%$ less likely to stop breastfeeding than those with $2^{\text {nd }}$ born babies (HR: 0.92; $\mathrm{p}<0.0001$ ). Unadjusted HR showed that operatively delivered mothers were $16 \%$ more likely to stop breastfeeding than mothers delivered vaginally (HR: 
1.16; $95 \% \mathrm{CI}, 1.01$ to 1.33 ) and IMD $3^{\text {rd }}$ quartile mothers were $22 \%$ less likely to stop breastfeeding than $1^{\text {st }}$ quartile mothers (HR: $0.78 ; 95 \%$ CI, 0.66 to 0.92 ). However these associations became insignificant after adjustment.

\section{Effects of in-hospital infant feeding practices on breastfeeding cessation}

Table 6 shows in-hospital infant feeding practices and their effects on breastfeeding cessation. KM analysis showed that there was no statistically significant difference between the duration in mothers who initiated breastfeeding within 1 hour after birth and those who initiated later. Giving babies infant formula and teats in hospital were significantly associated with breastfeeding duration. Mothers who gave infant formula to their babies breastfed for a mean duration of 17.7 weeks (median: 17 weeks) compared with 22.2 weeks (median: 27 weeks) for mothers who did not. The duration varied by the reasons for giving formula: those who gave formula based on informed choice had the shortest duration while those who gave because of medical indications breastfed longest. Mothers who gave teats to their babies breastfed for a mean duration of 17.2 weeks (median: 17 weeks) compared with 22.8 weeks (median: 27 weeks) for those who did not.

Unadjusted Cox regression results suggested that initiating breastfeeding after 1 hour post-delivery increased the likelihood of breastfeeding cessation but the association became insignificant after adjustment. Unadjusted HRs showed that mothers who gave infant formula while in hospital were $56 \%$ more likely to stop breastfeeding compared with those who did not (HR: 1.56; 95\% CI, 1.36 to 1.78 ). The association was slightly attenuated after adjustment (HR: 1.50; 95\% CI, 1.26 to 1.79). Adjusted sub-group analyses results also showed that mothers who gave infant formula based on informed choices were most likely to stop breastfeeding (HR: 1.91; 95\% CI, 1.49 to 2.44 ) while those who gave it based on medical indications were least likely to stop (HR: 1.34; $95 \% \mathrm{CI}, 1.09$ to 1.65$)$. A similar association was found for the use of teats in hospital. Unadjusted Cox regression results showed that mothers who gave teats while in hospital were $61 \%$ more likely to stop breastfeeding compared with those who did not (HR: $1.61 ; 95 \% \mathrm{CI}, 1.38$ to 1.87). The association was markedly attenuated after adjustment (HR: 1.26; 95\% CI, 1.04 to 1.53 ).

\section{Discussion}

Our study showed that ethnicity and parity were independent predictors of breastfeeding cessation. The UK 2005 Infant Feeding Survey and other studies have shown that White mothers breastfeed for shorter durations compared with mothers from other ethnic groups [3,21,37]. Among mothers from Asian background in the UK Pakistani and Bangladeshi mothers stop breastfeeding sooner than Indian mothers [23]. Many studies showed that breastfeeding duration increases with increasing parity $[3,26,38,39]$ and some evidence suggests that this might be related to previous breastfeeding experiences [3,22].

Table 6 Effects of in-hospital infant feeding practices on breastfeeding cessation.

\begin{tabular}{|c|c|c|c|c|c|c|}
\hline \multirow[t]{2}{*}{ Variable } & \multicolumn{2}{|c|}{ Breastfeeding duration from KM analyses } & \multirow{2}{*}{$\begin{array}{l}\text { Unadjusted Cox HR } \\
(95 \% \mathrm{Cl})\end{array}$} & \multirow[t]{2}{*}{$P$ value } & \multirow{2}{*}{$\begin{array}{c}\text { Adjusted Cox } \mathrm{HR}^{\Phi} \\
(95 \% \mathrm{Cl})\end{array}$} & \multirow[t]{2}{*}{$P$ value } \\
\hline & $\begin{array}{c}\text { Mean } \\
(95 \% \mathrm{Cl})\end{array}$ & $\begin{array}{l}\text { Median } \\
(95 \% \mathrm{Cl})\end{array}$ & & & & \\
\hline \multicolumn{7}{|l|}{ Breastfeeding initiation } \\
\hline Within $1 \mathrm{hr}$ & 22.2 (21.31 to 23.08$)$ & 27 (25.70 to 28.29) & $1^{\dagger}$ & - & - & - \\
\hline After $1 \mathrm{hr}$ & 20.3 (18.83 to 21.77$)$ & 24 (20.91 to 27.09) & 1.24 (1.08 to 1.42$)$ & 0.0028 & - & - \\
\hline - After 1 hr to 48 hrs & $20.3(18.95$ to 21.61$)$ & 25 (23.49 to 26.51$)$ & $1.20(1.04$ to 1.39$)$ & 0.0128 & - & - \\
\hline - After 48 hrs & 18.0 (13.43 to 22.57$)$ & 17 (8.40 to 25.59$)$ & $1.52(1.07$ to 2.17$)$ & 0.0195 & - & - \\
\hline \multicolumn{7}{|l|}{ Formula in hospital } \\
\hline No formula & 22.8 (21.86 to 23.68$)$ & 27 (26.08 to 27.92) & $1^{\dagger}$ & - & $1^{\dagger}$ & - \\
\hline Formula & $17.7(16.53$ to 18.89$)$ & 17 (13.94 to 20.06) & 1.56 (1.36 to 1.78$)$ & $<0.0001$ & $1.50(1.26$ to 1.79$)$ & $<0.0001$ \\
\hline - Yes (informed choice) & 19.7 (17.56 to 21.87$)$ & 20 (14.40 to 25.59$)$ & 1.73 (1.44 to 2.07$)$ & $<0.0001$ & 1.91 (1.49 to 2.44$)$ & $<0.0001$ \\
\hline - Yes (medical indication) & 16.6 (14.93 to 18.18$)$ & 17 (13.79 to 20.20$)$ & 1.38 (1.14 to 1.68$)$ & 0.0009 & 1.34 (1.09 to 1.65$)$ & 0.0058 \\
\hline - Yes (other reasons) & 17.0 (14.54 to 19.50$)$ & 18 (9.31 to 26.39 ) & 1.62 (1.20 to 2.18$)$ & 0.0016 & 1.53 (1.07 to 2.17 ) & 0.0181 \\
\hline \multicolumn{7}{|l|}{ Teats in Hospital } \\
\hline No teats & 22.4 (21.56 to 23.26$)$ & 27 (26.17 to 27.82 ) & $1^{\dagger}$ & - & $1^{\dagger}$ & - \\
\hline Yes & $17.2(15.88$ to 18.51$)$ & 17 (14.42 to 19.58$)$ & 1.61 (1.38 to 1.87$)$ & $<0.0001$ & 1.26 (1.04 to 1.53$)$ & 0.0203 \\
\hline - Yes (informed choice) & $17.3(15.52$ to 19.14$)$ & 18 (13.65 to 22.35) & 1.51 (1.23 to 1.87$)$ & 0.0001 & 1.01 (0.77 to 1.33 ) & 0.9231 \\
\hline - Yes (other reasons) & $17.2(15.35$ to 19.05$)$ & 17 (13.94 to 20.05) & 1.69 (1.39 to 2.06$)$ & $<0.0001$ & $1.32(1.04$ to 1.68$)$ & 0.0221 \\
\hline
\end{tabular}

\footnotetext{
$\Phi$ Variables that did not significantly contribute to the fitness of the model were dropped hence no adjusted HRs were calculated for them.
}

${ }^{\dagger}$ Reference category. 
Our study also found that giving infant formula and/ or teats to babies in hospital were significantly associated with breastfeeding cessation. These in-hospital infant feeding practices, especially when based on informed choices, may not in themselves be predictive of breastfeeding cessation but may be the early manifestations of maternal intentions relating to long-term infant feeding practices. The literature is consistent about the detrimental effects of using teats on breastfeeding outcomes [13,29-33] for which there may be a physiological explanation. The innate sucking reflex of the infant is satisfied by the teat, decreasing or eliminating the desire for contact with the nipple leading to reduction in breast milk production [13,30]. However others have suggested that teat use may simply be a marker for socio-demographic, psychosocial and cultural factors that determine both teat use and breastfeeding [30].

Our results suggested that the reasons for giving formula and/or teats may influence breastfeeding duration. Mothers who gave formula based on informed choice were most likely to stop breastfeeding. The timing of the decision to breastfeed influences breastfeeding duration [24] and it is likely this sub-group of mothers had not decided to breastfeed their babies early during the antenatal period. Those who gave formula for medical indications were least likely to stop breastfeeding and this was consistent with the WHO findings [13].

We found in our study that known predictors of breastfeeding cessation such as marital status, socio-economic deprivation, mode of delivery, and the timing of breastfeeding initiation, were not associated with breastfeeding cessation suggesting the peer support these women received might have been beneficial in attenuating the effects of these factors. Partnered mothers are usually more likely to breastfeed longer than single mothers [24-28] and in the developed countries affluent mothers tend to breastfeed longer [3,4,24,29]. Caesarean delivery is a recognised risk factor for breastfeeding initiation failure but there is no consistent evidence on its effect on breastfeeding duration [24,25]. Recent evidence has suggested that operative deliveries may have detrimental effects on breastfeeding outcomes [40-43] probably mediated by delayed breastfeeding initiation $[40,44]$ as a possible consequence of anaesthesia and analgesia [45,46]. However the UK 2005 Infant Feeding Survey found that mothers were equally likely to be breastfeeding at one and two weeks regardless of delivery methods [3]. Our study has found that mode of delivery was not associated with breastfeeding cessation supporting the conclusion by some that if the cultural and hospital environments generally promote breastfeeding, operative deliveries have little negative impact on breastfeeding outcomes [47].
Delayed breastfeeding initiation adversely affects breastfeeding duration and this formed the basis for the WHO/UNICEF recommendation that breastfeeding should be initiated within 30 minutes of birth even though it was admitted it is difficult to make exact recommendation about the timing of initiation [13]. In our study $77.7 \%$ of the mothers had initiated breastfeeding within one hour after delivery broadly in keeping with the recommendation but this was not associated with breastfeeding cessation.

\section{Implications}

Our study is one of the few to examine the effects of maternal factors and early infant feeding practices in hospital on breastfeeding in a peer support setting in the UK. The results have suggested that infant feeding practices associated with maternal ethnicity and parity may be more difficult to influence by peer support interventions. Peer support programmes, particularly those in multi-ethnic settings, will need to identify the needs of their various client groups in order to appropriately support them breastfeed longer. This could be done through an effective evaluation of such programmes.

We have also identified that in-hospital infant feeding practices which are likely to continue after leaving hospital have a negative impact on breastfeeding continuation. Infant feeding practices are influenced by the socio-cultural milieu and maternal intentions relating to these practices tend to be consolidated during the antenatal period. For peer support programmes to be more effective we suggest their support start during this period to enable mothers form healthier infant feeding intentions.

\section{Strengths, limitations and biases}

In this study we used a routinely collected data. Problems of a routine data source are largely related to completeness and accuracy. Missing dates of breastfeeding cessation affected BwD more than Hyndburn. Missing data resulted in 2,074 records out of 2,107 to be included in the final Cox multiple regression model. These made the study prone to selection bias. A potential confounder not included in the analyses was maternal age because this was not collected in many cases. An additional shortcoming relates to the accuracy of the estimated breastfeeding duration based on the on selfreported dates of breastfeeding cessation and infant feeding methods.

The strength of our study lies in the statistical methods used. The use of survival analysis to determine the breastfeeding duration provides a good understanding of breastfeeding behaviour over time [27]. Furthermore this type of analysis is suitable for data collected from follow-up studies that involve censoring and/or in which the time to an event is regarded as the outcome variable of interest [36]. To quantify the association between the 
factors and breastfeeding cessation Cox proportional hazards model was used. This model allows joint estimation of the effects of predictor variables on the risk for breastfeeding cessation rather than the duration itself [27].

\section{Conclusion}

In this study ethnicity, parity and in-hospital infant feeding practices remained independent predictors of breastfeeding cessation in this peer support setting. However other recognised predictors of breastfeeding cessation such as marital status, mode of delivery, and socio-economic deprivation were not found to be associated with breastfeeding cessation.

\section{Acknowledgements}

The authors will like to express their sincere gratitude to the directors, peer support workers and secretarial staff of Little Angels for their diligence and dedication in collecting and inputting a high quality data used for the study. The authors also acknowledge with gratitude the statistical advice provided by Dr. Islay Gemmell of the University of Manchester.

\section{Author details}

${ }^{1} \mathrm{NHS}$ Blackpool, Blackpool Stadium, Seasiders Way, Blackpool, FY1 6JX, UK. ${ }^{2}$ NHS Tameside and Glossop, New Century House, Windmill Lane, Denton, M34 2GP, UK. ${ }^{3}$ Little Angels, 13-15 Railway Road, Darwen, BB3 2RG, UK. ${ }^{4}$ Manchester Urban Collaboration on Health, Manchester Academic Health Sciences Centre, Stopford Building, University of Manchester, Manchester, M13 9PT, UK.

\section{Authors' contributions}

All the authors contributed to the various stages of this study. EM conceived the idea and liaised with Little Angels. EJ designed the data collection tool and supervised the data collection. GA did the analysis and wrote the paper with AV. All the authors read and commented on the drafts and approved of the final version for submission.

\section{Competing interests}

GA was involved in evaluating Little Angels peer support for breastfeeding mothers in Hyndburn. EJ is one of the Directors of Little Angels. EM was the acting Director of Public Health at Hyndburn and Ribble Valley PCT (now part of NHS East Lancashire) at the time of this study. Little Angels is funded in part by NHS East Lancashire and Blackburn with Darwen Borough Council.

Received: 24 November 2008

Accepted: 27 January 2010 Published: 27 January 2010

\section{References}

1. Dyson L, Renfrew M, McFadden A, McCormick F, Herbert G, Thomas J: Promotion of breastfeeding initiation and duration: Evidence into practice briefing London, NICE 2006

2. World Health Organization: The European health report 2005: public health action for healthier children and populations Geneva, WHO 2005.

3. Bolling K, Grant C, Hamlyn B, Thornton A: Infant Feeding Survey 2005 London, The Information Centre 2007.

4. Britton C, McCormick FM, Renfrew MJ, Wade A, King SE: Support for breastfeeding mothers. Cochrane Database Syst Rev 2007, CD001141.

5. Department of Health: Improvement, Expansion and Reform: The Next 3 Years. Priorities and Planning Framework 2003 - 2006 London, Department of Health 2002

6. Department of Health: Choosing Health: Making healthier choices London, Department of Health 2004.

7. Department of Health: National Service Framework for Children, Young People and Maternity Services London, Department of Health 2004.
8. Department of Health: Infant Feeding Recommendation London, Department of Health 2003.

9. Kramer S, Kakuma R: The optimal duration of exclusive feeding: A systematic review. Cochrane Database Syst Rev 2002, CD003517.

10. World Health Organisation: The optimal duration of exclusive breastfeeding: report on an expert consultation Geneva, WHO 2001.

11. BBC: 'Right to breastfeed' law planned London, BBC 2007http://news.bbc.co. uk/1/hi/uk_politics/6745445.stm, (accessed 20 Sept 2007).

12. Bartington S, Griffiths $\amalg$, Tate AR, Dezateux C, the Millennium Cohort Study Child Health Group: Are breastfeeding rates higher among mothers delivering in Baby Friendly accredited maternity units in the UK? Int J Epidemiol 2006, 35:1178-1186

13. World Health Organisation: Evidence for the Ten Steps to Successful Breastfeeding. WHO/CHD/98.9 Geneva, WHO 1998.

14. Henry S, Dykes F, Byrom S, Atkin M, Jackson E: Supporting Breastfeeding: Midwives Facilitating a Community Model. Essential Midwifery Practice Oxford, Blackwell Publishing LtdEdwards G, Byrom S 2007, 185-208.

15. All Party Parliamentary Group on Maternity: APPG Maternity Summer Reception 2007 - 11th July 2007 2007http://appg-maternity.org.uk/12.html, (accessed 23 Sept 2007).

16. Shribman S: Children's health, our future: A review of progress against the National Service Framework for Children, Young People and Maternity Services 2004 London, Department of Health 2007.

17. Ingram J, Rosser J, Jackson D: Breastfeeding peer supporters and a community support group: evaluating their effectiveness. Matern Child Nutr 2005, 1:111-118.

18. Kistin N, Abramson R, Dublin P: Effect of Peer Counselors on Breastfeeding Initiation, Exclusivity, and Duration Among Low-income Urban Women. J Hum Lact 1994, 10:11-15.

19. Sikorski J, Renfrew MJ, Pindoria S, Wade A: Support for breastfeeding mothers: a systematic review. Paediatr Perinat Epidemiol 2003, 17:407-417.

20. Yngve A, Sjöström M: Breastfeeding determinants and a suggested framework for action in Europe. Public Health Nutr 2001, 4:729-739.

21. Kelly YJ, Watt RG, Nazroo JY: Racial/Ethnic Differences in Breastfeeding Initiation and Continuation in the United Kingdom and Comparison With Findings in the United States. Pediatrics 2006, 118:e1428-e1435.

22. Novotny R, Hla MM, Kieffer EC, Park CB, Mor J, Thiele M: Breastfeeding Duration in a Multiethnic Population in Hawaii. Birth 2000, 27:91-96.

23. Thomas M: Infant feeding in Asian families: early practices and growth. Social Survey Report No.1366 Social Survey Report No. 1366 Basingstoke, Palgrave Macmillan 1997.

24. Dennis CL: Breastfeeding Initiation and Duration: A 1990-2000 Literature Review. J Obstet Gynecol Neonatal Nurs 2002, 31:12-32.

25. Forster DA, McLachlan HL, Lumley J: Factors associated with breastfeeding at six months postpartum in a group of Australian women. Int Breastfeed J 2006, 1.

26. Lande B, Andersen LF, Bærug A, Trygg KU, Lund-Larsen K, Veierød MB, Aa Bjørneboe G-E: Infant feeding practices and associated factors in the first six months of life: The Norwegian Infant Nutrition Survey. Acta Paediatr 2003, 92:152-161.

27. Scott JA, Binns CW, Oddy WH, Graham Kl: Predictors of Breastfeeding Duration: Evidence From a Cohort Study. Pediatrics 2006, 117:e646-e655.

28. Yeoh BH, Eastwood J, Phung H, Woolfenden S: Factors influencing breastfeeding rates in south-western Sydney. J Paediatr Child Health 2007, 43:249-255.

29. Wright CM, Parkinson $\mathrm{K}$, Scott J: Breast-feeding in a UK urban context: who breast-feeds, for how long and does it matter?. Public Health Nutr 2006, 9:686-691.

30. Callaghan A, Kendall G, Lock C, Mahony A, Payne J, Verrier L: Association between pacifier use and breast-feeding, sudden infant death syndrome, infection and dental malocclusion. Int J EB Healthcare 2005 3:147-167

31. Victora CG, Tomasi E, Olinto MT, Barros FC: Use of pacifiers and breastfeeding duration. Lancet 1993, 341:404-406.

32. Victora CG, Behague DP, Barros FC, Olinto MTA, Weiderpass E: Pacifier Use and Short Breastfeeding Duration: Cause, Consequence, or Coincidence? Pediatrics 1997, 99:445-453.

33. Vogel $A$, Hutchison BL, Mitchell EA: Factors associated with the duration of breastfeeding. Acta Paediatr 1999, 88:1320-1326.

34. Social Disadvantage Research Centre: Index of Multiple Deprivation London, Office of the Deputy Prime Minister 2004. 
35. World Health Organization: Indicators for assessing infant and young child feeding practices. Part 1 - Definitions. Geneva 2008http://www.emro.who.int/ cah/pdf/YYCF-Indicators-2007.pdf.

36. Woodward M: Modelling follow-up data. Epidemiology: Study design and data analysis Boca Raton, Chapman \& Hall/CRC 2004, 513-567.

37. Griffiths LJ, Tate AR, Dezateux C, the Millennium Cohort Study Child Health Group: The contribution of parental and community ethnicity to breastfeeding practices: evidence from the Millennium Cohort Study. Int J Epidemiol 2005, 34:1378-1386.

38. Bulk-Bunschoten AMW, Bodegom S, Reerink JD, Jong PP, Groot CJ: Reluctance to continue breastfeeding in The Netherlands. Acta Paediatr 2001, 90:1047-1053.

39. Taveras EM, Capra AM, Braveman PA, Jensvold NG, Escobar GJ, Lieu TA: Clinician Support and Psychosocial Risk Factors Associated With Breastfeeding Discontinuation. Pediatrics 2003, 112:108-115.

40. Chien LY, Tai CJ: Effect of Delivery Method and Timing of Breastfeeding Initiation on Breastfeeding Outcomes in Taiwan. Birth 2007, 34:123-130.

41. Leung GM, Lam T-H, Ho L-M: Breast-feeding and its relation to smoking and mode of delivery. Obstet Gynecol 2002, 99:785-794.

42. Patel R: Operative Delivery: Its association and subsequent maternal and infant morbidity - Analyses from the ALSPAC Cohort. The Research Findings Register Summary number 17322006.

43. Theofilogiannakou M, Skouroliakou M, Gounaris A, Panagiotakos D, Markantonis SL: Breast-feeding in Athens, Greece: factors associated with its initiation and duration. J Pediatr Gastroenterol Nutr 2006, 43:379-384.

44. Rowe-Murray HJ, Fisher JRW: Baby Friendly Hospital Practices: Cesarean Section is a Persistent Barrier to Early Initiation of Breastfeeding. Birth 2002, 29:124-131.

45. Forster DA, McLachlan HL: Breastfeeding Initiation and Birth Setting Practices: A Review of the Literature. J Midwifery Womens Health 2007, 52:1273-280

46. Rajan $\mathrm{L}$ : The impact of obstetric procedures and analgesia/anaesthesia during labour and delivery on breast feeding. Midwifery 1994, 10:87-103.

47. Kearney MH, Cronenwett LR, Reinhardt R: Cesarean Delivery and Breastfeeding Outcomes. Birth 1990, 17:97-103.

\section{Pre-publication history}

The pre-publication history for this paper can be accessed here:http://www. biomedcentral.com/1471-2431/10/3/prepub

doi:10.1186/1471-2431-10-3

Cite this article as: Agboado et al:: Factors associated with breastfeeding cessation in nursing mothers in a peer support programme in Eastern Lancashire. BMC Pediatrics 2010 10:3.

\section{Submit your next manuscript to BioMed Central and take full advantage of:}

- Convenient online submission

- Thorough peer review

- No space constraints or color figure charges

- Immediate publication on acceptance

- Inclusion in PubMed, CAS, Scopus and Google Scholar

- Research which is freely available for redistribution

Submit your manuscript at www.biomedcentral.com/submit
Ciomed Central 